\title{
Mitochondrial Disorders in Stroke and Chronic Brain Ischemia
}

\author{
Sergey Victorovich Kotov ${ }^{1}$, Olga Petrovna Sidorova ${ }^{2}$, Elena Vasilyevna Borodataya ${ }^{3}$, \\ Irina Anatolyevna Vasilenko ${ }^{4}$ \\ ${ }^{1}$ Department of Neurology, Moscow Regional Research and Clinical Institute («MONIKI»), Moscow, Russian Federation \\ ${ }^{2}$ Department of Neurology at the Moscow Regional Research and Clinical Institute («MONIKI»), Moscow, Russian Federation \\ ${ }^{3}$ Laboratory assistant at the faculty of advanced medical training Moscow Regional Research and Clinical Institute («MONIKI»), Moscow, \\ Russian Federation \\ ${ }^{4}$ Scientific Laboratory, Moscow Regional Research and Clinical Institute («MONIKI»), Moscow, Russian Federation
}

Email address:

kotovsv@yandex.ru (S. V. Kotov), sidorovaop2019@mail.ru(O. P. Sidorova), elena.borodataya@gmail.com (E. V. Borodataya), vasilenko0604@gmail.com (I. A. Vasilenko)

\section{To cite this article:}

Sergey Victorovich Kotov, Olga Petrovna Sidorova, Elena Vasilyevna Borodataya, Irina Anatolyevna Vasilenko. Mitochondrial Disorders in Stroke and Chronic Brain Ischemia. American Journal of Psychiatry and Neuroscience. Vol. 9, No. 2, 2021, pp. 68-76.

doi: 10.11648/j.ajpn.20210902.17

Received: April 7, 2021; Accepted: April 21, 2021; Published: May 31, 2021

\begin{abstract}
Stroke is the leading cause of disability and death in the adult population. Modern methods of treating patients with acute ischemic stroke include thrombolytic therapy with a narrow therapeutic window and endovascular thrombectomy. The development of other methods of treatment of brain hypoxia in the penumbra zone is relevant. Mitochondria, which are involved in immediate and delayed molecular mechanisms of adaptation to hypoxic stress in the cerebral cortex, primarily respond to hypoxia. Hypoxia induces reprogramming of the mitochondrial respiratory chain function and switching from oxidation of substrates of the respiratory chain complex I to succinate oxidation (complex II). The brain's need for succinate increases. Clinical studies have shown a positive effect of drugs containing succinates on the course of stroke. The study of mitochondrial function is carried out mainly in an experiment. In the present study of mitochondrial disorders in stroke and chronic brain ischemia in adult patients, the quantitative method proposed by A. G. Pearse was used to assess the activity of mitochondrial enzymes of peripheral blood lymphocytes, which are referred to as the "enematic mirror" of tissues. In acute cerebral ischemia, a compensatory increase in the activity of succinate dehydrogenase was observed on the first day, indicating the tension (increased activity) of the second complex of the mitochondrial respiratory chain.. These data confirm the need to prescribe succinic acid in the acute phase of stroke. At the same time, the dose of $250 \mathrm{mg}$ per day is not sufficient for patients with increased body weight. The standard dose of the drug should be higher, taking into account the different body weight of patients. In patients with stroke, there was also a decrease in the activity of $\alpha$-glycerophosphate dehydrogenase, which is involved in the fat metabolism of mitochondria, which is an indication for the appointment of carnitine. In chronic brain ischemia, the activity of succinate dehydrogenase and $\alpha$-glycerophosphate dehydrogenase decreased, indicating indications for the appointment of idebenone and carnitine along with vasodilator therapy and endovascular thrombectomy. Thus, the results of a study of mitochondrial function in patients with acute and chronic brain ischemia are presented. Violations of complex II in the respiratory chain cycle and violation of fat metabolism were revealed, indicating indications for the appointment of energotropic therapy.
\end{abstract}

Keywords: Stroke, Chronic Brain Ischemia, Mitochondria, Succinate Dehydrogenase, $\alpha$-glycerophosphate Dehydrogenase

\section{Introduction}

Stroke is the leading cause of disability and death in the adult population. Modern advanced treatment methods for patients with acute ischemic stroke include thrombolytic therapy and endovascular thrombectomy. However, only a 
limited number of patients receive this treatment, since the therapeutic window is narrow. Therefore, the development of other methods for treating brain hypoxia in the penumbra zone is relevant. It is known that mitochondria - cellular organelles that are the energy stations of the cell-primarily react to hypoxia. This cellular bioenergetic apparatus is involved in the development of immediate and delayed molecular mechanisms of adaptation to hypoxic stress in the cerebral cortex. Hypoxia induces reprogramming of the mitochondrial respiratory chain function and switching from oxidation of substrates of the respiratory chain complex I to succinate oxidation (complex II) (Figure 1) [1]. Transient, reversible, compensatory activation of the respiratory chain complex II is the main mechanism of direct adaptation to hypoxia, which is necessary for (1) succinate-bound energy synthesis in conditions of oxygen deficiency and the formation of urgent resistance in the body; (2) succinaterelated stabilization of HIF-1A and initiation of its transcriptional activity associated with the formation of long- term adaptation; (3) succinate-related activation of the GPR91 succinate-specific receptor [2-5]. It has been shown that in conditions of normal oxygen supply to body tissues, the succinate-dependent GPR91 receptor is present in various amounts in all aerobic tissues, except for red blood cells. The maximum density of this receptor was observed in the myocardium. Hypoxic expression of GPR91 is tissue-specific and depends on the duration and severity of hypoxia. It is maximal in the cerebral cortex. A single hypoxic exposure induces immediate expression of GPR91 in the cerebral cortex for 15-60 minutes, which correlates with the development of urgent tolerance of the body to hypoxia [4]. Hypoxia-induced direct expression of HIF-1A transcription factor in the cerebral cortex is regulated by succinate and induced by medicines containing succinate [3]. These data indicate an increased need of the brain for succinate in hypoxia. Clinical studies have shown a positive effect of drugs containing succinates on the course of stroke [6-11].

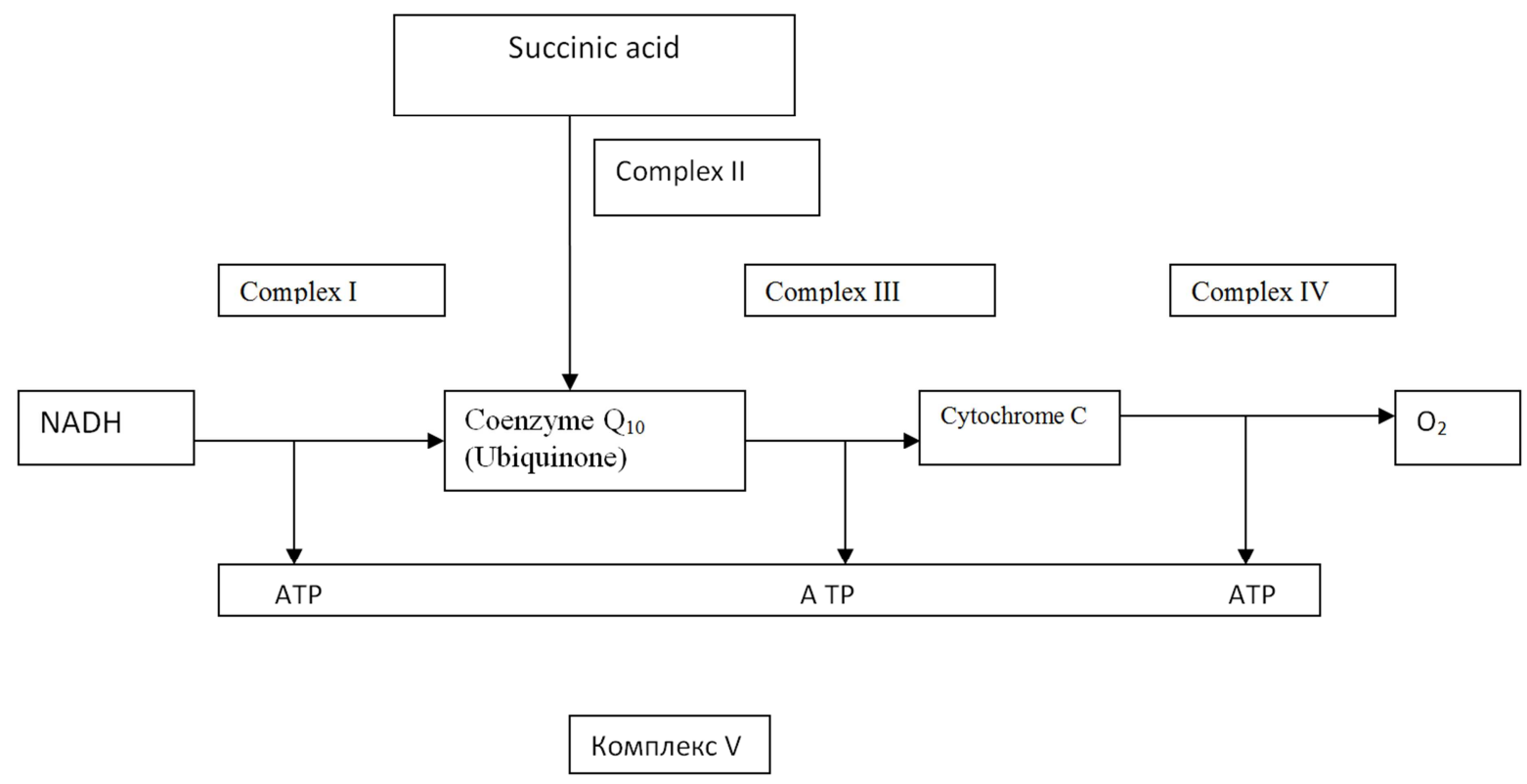

Figure 1. Complexes of the electron transport chain in mitochondria.

In ischemic stroke, the blood-brain barrier is disrupted, which is regulated by mitochondria, immune cells, cytokines, and adhesion proteins [12]. Mitochondria play not only a bioenergetic role, but are also important in a wide range of cellular functions and signaling events [13]. Hayakawa К. и др. (2016) [14] showed that transient focal cerebral ischemia in mice promotes the penetration of astrocytic mitochondria into neighboring neurons and increases cell survival. These results suggest a new mitochondrial mechanism of neuroglial connections, which may have a neuroprotective mechanism after stroke. It is assumed that cellular regenerative medicine, in particular stem cell transplantation, may be promising in stroke, since the transplanted cells and their components can repeat the growth and function of the neurovascular unit, which plays a major role in recovery after a stroke. Since mitochondria are important in energy metabolism and stress response, stem cells will be a source of healthy mitochondria and will contribute to the recovery of damaged brain after stroke [15].

Thus, mitochondrial disorders play an important role in the pathogenesis of brain ischemia. The study of mitochondrial function in stroke and chronic brain ischemia is relevant, will allow us to study the pathogenesis of stroke and chronic brain ischemia in depth, and develop new approaches to treatment.

The purpose of this study is to study mitochondrial disorders in the pathogenesis of acute and chronic brain ischemia in patients to improve the effectiveness of care for this pathology.

\section{Materials and Methods}

To assess tissue respiration (mitochondrial respiratory 
chain) and other types of metabolism in mitochondria, cytochemical analysis of the activity of mitochondrial enzymes of peripheral blood lymphocytes was performed. We examined 22 adult patients with acute and chronic brain ischemia. Of these, 7 patients were examined in the acute phase of ischemic stroke. Two of these patients were reexamined for mitochondrial function a week late. The group of patients with chronic cerebral ischemia included 15 patients. 30 healthy adult subjects were examined as a control group. The activity of 4 mitochondrial enzymes was evaluated: (1) participating in complex II of the mitochondrial respiratory chain (succinate dehydrogenase, $\mathrm{SDH}) ;(2)$ in the exchange of fatty acids ( $\alpha$-glycerophosphate dehydrogenase, $\alpha-$ GFDH); (3) in carbohydrate metabolism (lactate dehydrogenase, LDH); (4) in the exchange of amino acids (glutamate dehydrogenase, GDH).

For the cytochemical study of the activity of mitochondrial enzymes in peripheral blood lymphocytes, the method proposed by A. G. E. Pearse in the modification of R. P. narcissov was used [16]. Chemical reagents were used: fixative for peripheral blood smears (acetone-Trilon), cytochemical kits for determining the activity of dehydrogenases (LLC NPP "Polikom"), dye for finishing the nuclei (methyl green).

Equipment: binocular microscope (magnification not less than $10 * 40$ ), equipped with a water immersion lens, water thermostat that provides temperature maintenance with an accuracy of $0.1^{\circ} \mathrm{C}, \mathrm{PH}$ meter.

Method for quantitative cytochemical determination of enzyme activity in peripheral blood cells (formulation of reactions). Reactions are performed on blood smears prepared on fat-free slides. Smears are air-dried at room temperature for 10-15 minutes. The formulation of the reaction includes three stages: the fixation of smears, the reaction to detect the activity of enzymes, and the staining of the nuclei. Fixation of drugs is carried out in $60 \%$ acetone solution saturated with Trilon $\mathrm{B}$ at $\mathrm{pH}=5.2-5.4$ at room temperature for 30 - 40 seconds (for lymphocytes). After fixing, the preparations are washed with distilled water and dried at room temperature in the air. Composition of the incubation medium: for $40 \mathrm{ml}$ of phosphate buffer, $13 \mathrm{mg}$ of p-nitrotetrazolium violet, $13 \mathrm{mg}$ of Trilon $\mathrm{B}$, a specific substrate for a specific enzyme. The reaction is carried out at $\mathrm{pH}=7.3$ and a temperature of $37^{\circ} \mathrm{C}$ for 60 minutes in an aqueous thermostat. After incubation, the smears are washed with water and immersed in a saturated solution of methyl green (nuclear dye) for 15-20 seconds, after which the smears are washed again and dried at room temperature in air. The finished smears are microscopized under water immersion on a Micmed-6 microscope. The activity of the enzyme in the cell is judged by the number of dark purple formazan granules formed during the reduction of p-nitrotetrazolium violet (Figure 2). To determine the enzyme activity in the lymphocyte population, the number of granules in $30-100$ cells is counted. The enzymatic activity when using this method is expressed in granules / lymphocyte, which corresponds to the average number of granules of the cytochemical reaction product - formazan.

The lactate level was determined in whole heparinized blood by the amperometric, ensematic method using a substrate-specific electrode on a blood gas analyzer ABL800 FLEX.

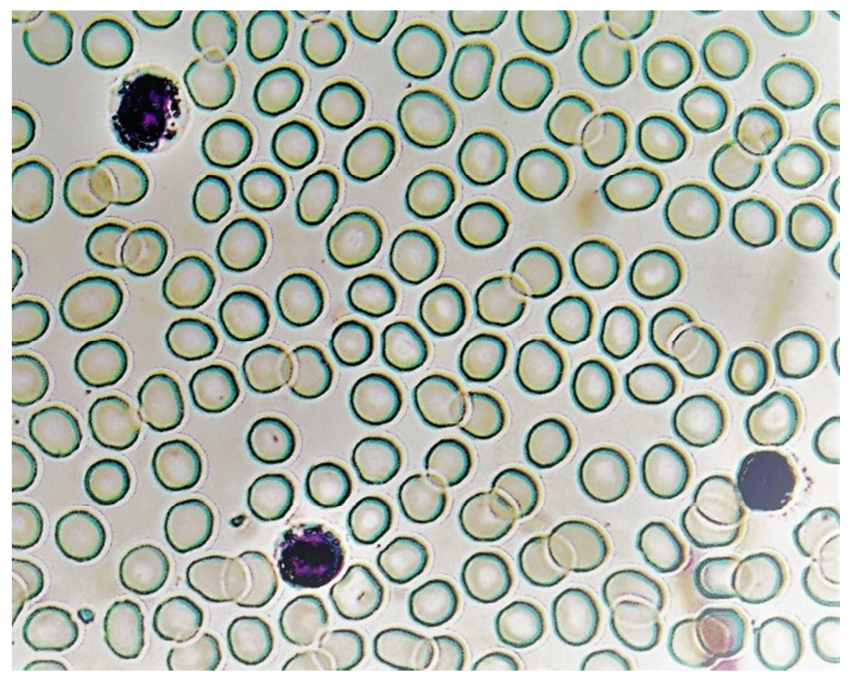

Figure 2. Revealing the activity of succinate dehydrogenase in lymphocytes by a quantitative cytochemical method (view under a microscope, magnification 600, dark granules according to the periphery of the cell production).

\section{Statistical Analysis}

Determined the median, the confidence interval of the median. The nonparametric Wilcoxon-Mann-Whitney test was used to determine the significance of the differences in the parameters in patients with the control group. The difference was considered statistically significant at $\mathrm{p}<0.05$.

\section{Results}

\subsection{Mitochondrial Disorders in Stroke}

7 patients with ischemic stroke at the age from 25 to 77 years were examined. The median age of the patients was 36 years. The confidence interval for the median was $16.2-55.7$ years. The onset of the disease before the age of 40 was in 4 patients and in 3 patients after 40 years. 5 women and 2 men were examined. Blood sampling was performed in the first 24 hours after the onset of the disease. The study was repeated in two patients after 7 days.

As can be seen from Table 1 and Figure 3 median SDH activity (II complex of the mitochondrial respiratory chain) increased in patients in the first 24 hours after the onset of ischemic stroke compared with control data $(p>0.05)$. The median activity of $\alpha-\mathrm{GPDH}$, which is involved in mitochondrial fat metabolism, was 7.7 granules / lymphocyte in patients (in the control group - 8.5 granules / lymphocyte) ( $>0.05$ ). The median GDH activity in patients was 9.6 granules / lymphocyte (in the control group - 8.8) ( $\mathrm{p}>0.05)$. The median index of LDH activity in patients was 15.3 granules / lymphocyte (in the control group - 15.5 granules / lymphocyte) $(\mathrm{p}>0.05)$. 

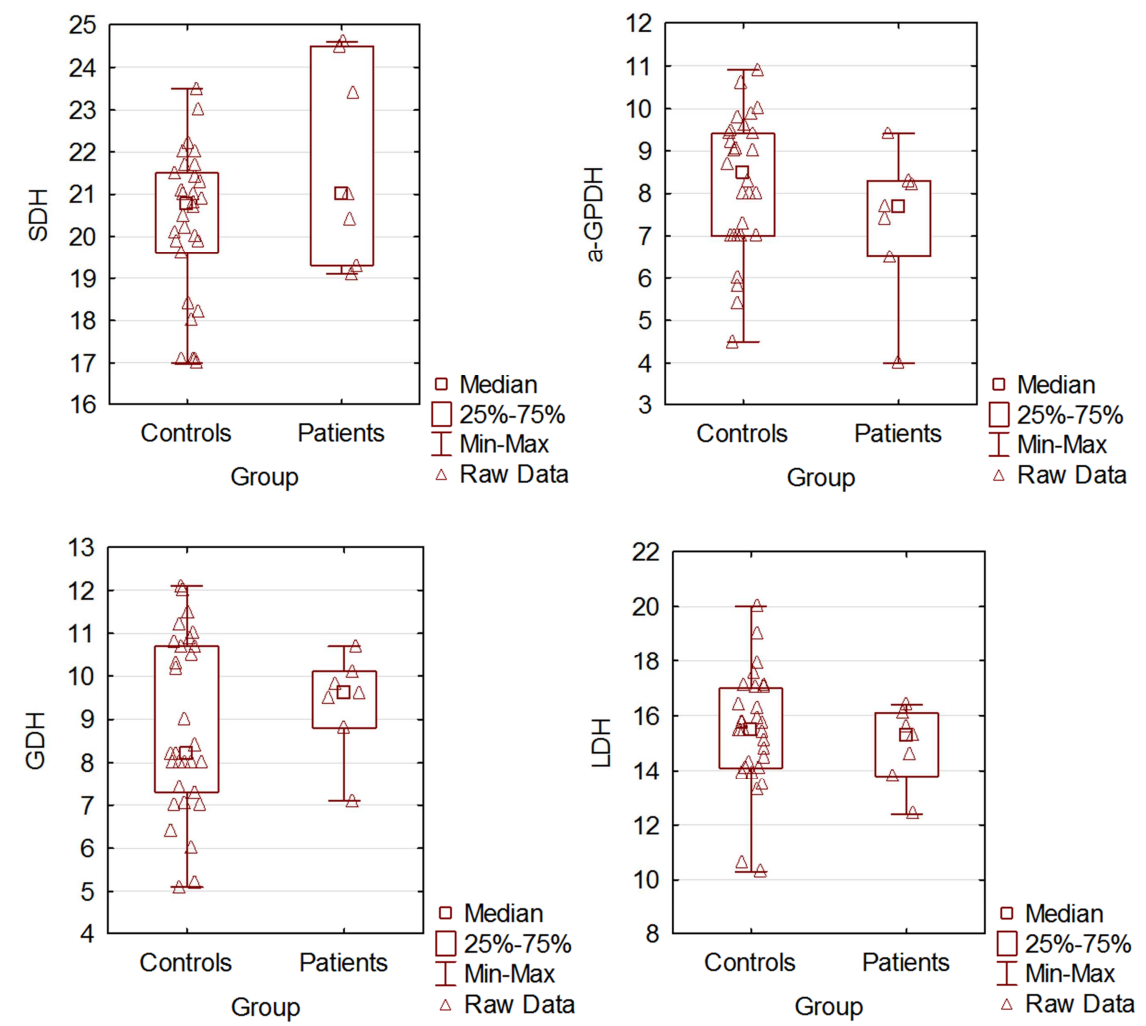

Figure 3. Quantitative assessment of the activity of mitochondrial enzymes in peripheral blood lymphocytes on the first day of ischemic stroke. Horizontal the examined groups, vertical - the median of the activity of mitochondrial enzymes of peripheral blood lymphocytes (granules / lymphocyte).

Table 1. Indicators of mitochondrial enzyme activity in peripheral blood lymphocytes in patients with acute ischemic stroke (granules /lymphocyte),

\begin{tabular}{lllllll}
\hline $\mathbf{N}$ & Gender & Age & SDH & $\boldsymbol{\alpha}$-GPDH & GDH & LDH \\
\hline 1 & Man & 26 & 23,4 & 4,0 & 7,1 & 12,4 \\
2 & Woman & 29 & 24,5 & 6,5 & 9,8 & 13,8 \\
3 & Woman & 25 & 24,6 & 8,3 & 10,7 & 16,4 \\
4 & Woman & 68 & 20,4 & 9,4 & 8,8 & 15,3 \\
5 & Woman & 36 & 19,1 & 7,4 & 9,6 & 15,6 \\
6 & Woman & 54 & 19,3 & 7,7 & 9,5 & 16,1 \\
7 & Man & 77 & 23,4 & 8,2 & 10,1 & 14,6 \\
Median in the patient group & & 7,7 & 9,6 & 15,3 \\
Confidence interval for the median in the group of patients & $21,1-25,7$ & $6,1-9,2$ & $9,1-11,0$ & $14,0-16,6$ \\
\multicolumn{2}{l}{ Median in the control group } \\
\multicolumn{2}{l}{ Confidence interval for the median in the control group } & 20,75 & 8,5 & 15,5 \\
\hline
\end{tabular}

In two patients with ischemic stroke, a repeated determination of the activity of mitochondrial enzymes was carried out 1 week after the first blood sampling. Table 2 presents data on the dynamics of indicators of the level of mitochondrial enzymes in two patients (overweight - patient 1 and underweight - patient 2) against the background of the administration of the same dose of succinic acid preparation (2nd complex of the mitochondrial respiratory chain) (5\% Mexidol 5.0 solution). A week after the onset of stroke in the 1st patient with increased body weight against the background of the administration of a drug containing succinate at a dose of $250 \mathrm{mg}$ per day, a decrease in SDH activity was noted below the reference values, which probably indicates that the dose of the drug was insufficient for this patient and, as a consequence of this, a decrease in the activity of the 2nd complex of the mitochondrial respiratory chain, its depletion. In the second patient with low body weight, on the contrary, this indicator increased (19.3 to 20.6 granules / lymphocyte). Which indicates a sufficient dose of the drug for this patient.

The $\alpha$-GPDH activity in both patients was lower than the reference values on the first day of the disease $(7.7$ and $8.2 \mathrm{~g}$ / lymphocyte in patients, the reference values were 9.0-12.0 g / lymphocyte). A week later, the first patient's indicator increased to 8.0. And in the second patient it dropped to 7.8. The level of GDH activity was within the normal range in both patients on the first day of stroke (9.5 and 10.1 granules / lymphocyte in patients (reference values in the control group - 9.0-12.0 granules / lymphocyte). A week later, in the first patient with increased body weight, the enzyme activity decreased. In a patient with low body weight, the enzyme activity remained at the same level. $\mathrm{LDH}$ activity was within 
the normal range in both studied patients on the first day and one week after the onset of the disease. In the first patient, the enzyme activity decreased within normal values from 16.1 granules / lymphocyte on the first day to 14.0 granules / lymphocyte in a week. In the second patient, the enzyme activity increased within the normal range from $14.6 \mathrm{~g} /$ lymphocyte to 16.8 granules / lymphocyte, with reference values of 10.0-17.0 granules / lymphocyte).

Table 2. Dynamics of indicators of the activity of mitochondrial enzymes in peripheral blood lymphocytes in patients with ischemic stroke (granules / lymphocyte) on the 1 st and 7 th days of the disease.

\begin{tabular}{|c|c|c|c|c|c|c|c|c|c|c|}
\hline $\mathbf{N}$ & Gender & Age & SDH & & $\alpha-$ GPDH & & GDH & & LDH & \\
\hline & & & On the 1st day & In 7 days & On the 1st day & In 7 days & On the 1st day & In 7 days & On the 1st day & In 7 days \\
\hline 1 & Woman & 52 & 19,1 & 17,4 & 7,7 & 8,0 & 9,5 & 8,7 & 16,1 & 14,0 \\
\hline 2 & Man & 77 & 19,3 & 20,6 & 8,2 & 7,8 & 10,1 & 10,1 & 14,6 & 16,8 \\
\hline
\end{tabular}

\subsection{Mitochondrial Disorders in Chronic Cerebral Ischemia}

We examined 15 patients with chronic cerebral ischemia. The median age of the patients was 55.0 years. The median confidence interval is $18.2-61.8$ years. 10 women and 5 men were examined.
Mitochondrial dysfunction was found in all patients. SDH activity was changed in 14 out of 15 patients $(93.3 \%)$. The median activity of the enzyme in patients was 19.2 granules / lymphocytes (in the control group - 20.75 ggranules / lymphocyte, $\mathrm{p}<0.05$ ) (table 3 ).

Table 3 Activity of mitochondrial enzymes (granules / lymphocyte) in peripheral blood lymphocytes in chronic cerebral ischemia.

\begin{tabular}{|c|c|c|c|}
\hline Mitochondrial enzymes & \multirow{2}{*}{ Median } & \multirow{2}{*}{ Confidence interval for the median } & \multirow{2}{*}{$\mathbf{p}$} \\
\hline SDH & & & \\
\hline Group of patients with chronic cerebral ischemia $(\mathrm{N}=15)$ & 19,2 & $17,87-20,53$ & $<0,05^{*}$ \\
\hline $\begin{array}{l}\text { Group of healthy persons }(\mathrm{N}=30) \\
\alpha \text {-GPDH }\end{array}$ & 20,75 & $19,95-21,55$ & \\
\hline Group of patients with chronic cerebral ischemia $(\mathrm{N}=15)$ & 7,0 & $6,05-7,95$ & $<0,05^{*}$ \\
\hline $\begin{array}{l}\text { Group of healthy persons }(\mathrm{N}=30) \\
\text { GDH }\end{array}$ & 8,5 & $7,79-9,21$ & \\
\hline $\begin{array}{l}\text { Group of healthy persons }(\mathrm{N}=30) \\
\text { LDH }\end{array}$ & 8,8 & $7,89-9,71$ & \\
\hline Group of patients with chronic cerebral ischemia $(\mathrm{N}=15)$ & 13,5 & $11,47-15,53$ & $<0,05^{*}$ \\
\hline Group of healthy persons $(\mathrm{N}=30)$ & 15,5 & $14,57-16,43$ & \\
\hline
\end{tabular}

Note: * - difference in comparison with the control group of healthy individuals.

In $86.7 \%$ (13 out of 15$)$ cases, a decrease in the activity of the $\alpha$-GPDH enzyme involved in mitochondrial fat metabolism was revealed. The median enzyme activity in patients was 7.0 granules / lymphocyte (in the control group 8.5 granules / lymphocyte, $\mathrm{p}<0.05)$.

In $73.3 \%$ (11 out of 15 ) patients, a decrease in the activity of GDH, which is involved in the exchange of amino acids, was revealed. The median of the indicator was 6.6 granules / lymphocyte (in the control group - 8.8 granules / lymphocyte; $\mathrm{p}<0.05)$.

The median LDH activity, reflecting carbohydrate metabolism, was reduced in comparison with the control data (13.5 granules / lymphocyte in patients, 15.5 granules / lymphocyte in the control group, $\mathrm{p}<0.05$ ).

The level of lactate in the blood before meals was increased in patients with chronic cerebral ischemia. The median was $2.6 \mathrm{mmol} / \mathrm{L}$ before carbohydrate loading. After carbohydrate loading, the median blood lactate level was 2.4 $\mathrm{mmol} / \mathrm{L}$, with a normal value of up to $2.2 \mathrm{mmol} / \mathrm{L}$.

\section{Discussion}

In the presented material, a technique was used for the quantitative assessment of the activity of mitochondrial enzymes in peripheral blood lymphocytes, which allows one to objectively assess all 4 types of metabolism in mitochondria in patients (respiratory chain cycle, fat metabolism, amino acid and carbohydrate metabolism). In acute cerebral ischemia in the first 24 hours from the onset of the disease, a compensatory increase in the activity of SDH was noted, indicating the tension (increased activity) of the II complex of the mitochondrial respiratory chain. These data confirm the necessity and expediency of prescribing succinic acid preparations in the acute phase of stroke. It has been shown that the dose of the drug $250 \mathrm{mg}$ per day is not sufficient for patients with increased body weight. The standard dose of the drug should be more than $250 \mathrm{mg}$ of succinate, taking into account the different body weights of patients. In patients with stroke, a decrease in the activity of $\alpha$-GPDG is also noted. Taking these data into account, it is possible that in the acute phase of a stroke (during the first day), another energotropic drug, carnitine, should also be prescribed. Subsequently, during the rehabilitation period, energotropic drugs can also be used, given the role of mitochondria in recovery from a stroke. But instead of succinic acid preparations, idebenone preparations (III complex of the respiratory chain) should be used subsequently, which are intended for long-term use, since they do not cause proliferative cell growth. Idebenone preparations, in contrast to the natural substance coenzyme 
Q10, have a short side chain and easily penetrate the bloodbrain barrier. You can also use carnitine preparations, since these drugs can be prescribed simultaneously, given that they are involved in various types of metabolism in the mitochondria. These energotropic medications also help restore cognitive functions that are impaired in stroke [25, 26].

With prolonged chronic cerebral ischemia, a statistically significant decrease in the indices of SDH activity was revealed, which indicates the decompensation of the II complex of the mitochondrial respiratory chain and the need for idebenone. A statistically significant decrease in the activity of $\alpha$-GPDH indicates indications for the prescription of carnitine preparations. Energotropic therapy is indicated along with vasodilator therapy and endovascular thrombectmia.

Thus, in the study of the function of mitochondria in the acute period of stroke in all examined patients, violations in the cycle of the respiratory chain were revealed, which is an indication for the appointment of succinic acid preparations. In most cases, disorders in the fat metabolism of mitochondria are also determined. This is an indication for the prescription of carnitine preparations. Chronic cerebral ischemia leads to a significant decrease in the activity of mitochondrial enzymes, including those involved in the cycle of the mitochondrial respiratory chain and the metabolism of fatty acids. What is the indication for the appointment of idebenone and carnitine preparations along with vasodilator therapy and endovascular thrombectmia.

Mitochondrial dysfunction is the main mechanism of the body's response to oxygen deficiency. In the life of a cell, the leading metabolic link is energy metabolism, which is necessary for the implementation of various energydependent functions (mechanical, osmotic, chemical, electrical). With a decrease in intracellular ATP by $10-15 \%$, signs of inhibition of energy-dependent processes appear [17] With a decrease in ATP by $25-30 \%$, they are completely suppressed. Energy metabolism is a target for hypoxia. The suppression of energy synthesis in the presence of oxygen deficiency leads to a decrease in the content of intracellular ATP below the physiological norm and the conjugate inhibition of energy-dependent processes. All this is the cause of multisystem, multiorgan functional and metabolic disorders that are characteristic of hypoxia. Aerobic oxidative processes occur in the respiratory chain of mitochondria - the main suppliers of energy. The main flow of oxygen is directed to the mitochondria. $80-90 \%$ of the oxygen entering the cells (neuron and cardiocytes) is consumed by the mitochondria.

The mitochondrial respiratory chain is a group of enzymes and proteins involved in oxidative phosphorylation and ATP production. They are found on the inner membrane of mitochondria and cristae. The enzymes of the mitochondrial respiratory chain are combined into complexes and perform the function of redox carriers of protons and electrons. There are four complexes of the mitochondrial electron transport chain: complex I (NADH: ubiquinone oxidoreductase); complex II (succinate (succinic acid): ubiquinone oxidoreductase); complex III (ubiquinol: ferrocytochrome coxidoreductase or cytochrome bc complex); complex IV (ferrocytochrome c: oxygen oxidoreductase or cytochrome c oxidase); complex V (synthesis of ATP). The released energy is used for the synthesis of ATP. ATP is an important accumulator of chemical energy, as energy is generated during its breakdown. A decrease in oxygen delivery to the cell leads to functional and metabolic disorders, among which a change in the complexes of the mitochondrial respiratory chain plays a major role. This process can be represented as follows:

1. the change in the complex of the respiratory chain begins at its substrate (NAD-dependent) site - the I complex of the respiratory chain. In response to a decrease in oxygen concentration, the activity of this complex of the respiratory chain is first increased and subsequently suppressed.

2. This process is accompanied by the inclusion of compensatory pathways for substrate oxidation, which allow maintaining the conjugated function of III and IV complexes, and activation of complex II of the respiratory chain (succinate oxidase oxidation pathway) plays the most important role (compensatory stage of tissue hypoxia).

3. An increase in the severity and duration of hypoxia extends to complex III of the respiratory chain (cytochrome bc region) (decompensation stage) and then to complex IV (cytochrome oxidase), which is inactivated only at low PO2 values (terminal stage of bioenergetic hypoxia). Consequently, in the process of increasing the severity of hypoxia, regulatory and limiting mechanisms change.

Mironova G. D. et al. (2019) [2] for the first time studied the mitochondrial structure and content of subunits (NDUFV2, SDHA, Cyt b, COX1) of mitochondrial respiratory complexes I-IV, as well as hypoxia-inducible factor (HIF-1 $\alpha$ ) in the cerebral cortex of rats, depending on the severity of hypoxia. They used various modes of 30minute hypobaric hypoxia (pO2 14, 10 and 8\%). At 14 and $10 \%$ hypoxia, animals developed compensatory structural and metabolic reactions aimed at maintaining the energy homeostasis of cells. At the same time, a decrease in the oxygen concentration in the inhaled air to $8 \%$ led to the appearance of destructive processes in the mitochondria of the brain.

Dysfunction of mitochondria contributes to the development of neurological disorders in chronic cerebral ischemia as a result of impaired oxidative phosphorylation (Zhao X. Y. et al., 2019)[18]. Neurons vary widely in shape, size, type of neurotransmitter, and number of synapses. Neurons are very sensitive to changes in oxygen concentration. The consequence of hypoxia is the launch of a series of biochemical reactions called the ischemic cascade. In fact, the ischemic cascade includes very complex processes that occur simultaneously and interact with each other. The key role in the responses of neurons to hypoxia is 
played by changes associated with mitochondria, which occur immediately after hypoxia at the beginning of the ischemic cascade. Disturbances in the functions of mitochondria are recognized as an important element not only in acute, but also in chronic hypoxia (Lenart J., 2017)[19]. The experiment showed that mitochondria isolated from the hippocampus of rats from the ischemic group had reduced levels of pyruvate dehydrogenase and increased oxidative stress, which was manifested in an increase in the production of hydrogen peroxide. Since mitochondrial deficiency of oxidative metabolism, namely mitochondrial bioenergetic deficiency, directly affects neuronal function, it may also contribute to the onset of vascular dementia (Du J. и соавт., 2017)[20]. These data indicate that such patients, along with vasodilator drugs, should be prescribed energotropic drugs to correct neurological symptoms and secondary cognitive disorders.

Kim A. Y. et al (2017) [21] studied the activity of glutamate dehydrogenase in cerebral ischemia. Glutamate dehydrogenase plays an important role in the metabolism of glutamate and plays a central role in expanding the pool of intermediate alpha-ketoglutarate of the tricarboxylic acid cycle, which improves overall bioenergetics. Under conditions of high energy demand, maintaining ATP production leads to the appearance of functionally active mitochondria. The authors induced cerebral ischemia in a mouse model. A glutamate dehydrogenase activator was used to stimulate the activity of glutamate dehydrogenase. As a result of its use, the level of extracellular glutamate decreased. Thus, mitochondrial glutamate dehydrogenase activators increase the production of alpha-ketoglutarate as an alternative energy source for use in the tricarboxylic acid cycle under energy depletion conditions. The results of the study showed that increased glutamate oxidation represents a new therapeutic intervention for cerebral hypoxia.

The severity of energy exchange disorders is determined using various methods. A high-resolution respirometry method is used, which allows highly accurate measurement of oxygen in a small amount of biological material. The rate of consumption and concentration of oxygen by living cells and tissues is measured with the maximum approximation of the measurement to in vivo. There is a polarographic method with measuring the amount of oxygen using a Clark electrode. At the same time, the rate of oxygen consumption, the rate of ATP synthesis and other indicators are determined. The membrane potential of mitochondria is being studied [22]. Such research methods are used mainly in experiments. In clinical practice, as well as in the development and assessment of the effectiveness of drugs, the state of mitochondrial function has not been studied enough. In clinical practice, it is promising to determine energy production in peripheral blood lymphocytes, which are referred to as the "enzyme mirror of tissues". A quantitative cytochemical method is used to study the activity of mitochondrial enzymes in peripheral blood lymphocytes, proposed by A. G. Pearse [16]. This method allows to investigate the activity of mitochondral enzymes intracellularly by the activity of dehydrogenases in peripheral blood leukocytes. In the presented study, the method proposed by A. G. Pearse. It made it possible to objectively assess the functional state of mitochondria, determine the need for energotropic therapy in acute and chronic cerebral ischemia.

For the correction of mitochondrial dysfunctions, drugs are successfully used, which include substances involved in tissue respiration, in the complexes of the mitochondrial respiratory chain $[23,24]$. Since the cause of acute and chronic cerebral ischemia is the occlusion of cerebral vessels, the main treatment method is to restore blood flow (thrombolysis and surgical treatment for acute ischemia, vasodilator therapy for chronic ischemia). Nevertheless, symptomatic therapy is important, which reduces the effects of hypoxia, including cognitive disorders. These drugs include energotropic drugs - substances whose effects are aimed at correcting mitochondrial dysfunction and restoring the energy synthesizing function of the cell. Considering that in acute hypoxia, the II complex of the respiratory chain (succinate oxidative oxidation pathway) is activated, which plays a major role in the compensatory stage of tissue hypoxia, with the development of a stroke, succinic acid preparations should be prescribed for 5-7 days. Subsequently, in stroke and chronic cerebral ischemia, it is advisable to prescribe preparations of coenzyme Q10 (III complex of the respiratory chain), cytochrome C (IV complex of the respiratory chain). The disadvantage of drugs containing cytochrome $\mathrm{C}$ is that they are prescribed only parenterally. Ko Coenzyme Q10 poorly penetrates the blood-brain barrier due to its long side chain (Figure 3 ). Coenzyme $Q_{10}$ poorly penetrates the blood-brain barrier due to its long side chain. Therefore, its synthetic analogue idebenone (coenzyme Q6) should be used. The idebenone molecule structurally resembles ubiquinone (Figure 1). Idebenone and ubiquinone have the same benzene ring, but idebenone has a shorter side chain. The side chain of the idebenone molecule is 5 times smaller than the ubiquinone molecule, which ensures its ability to penetrate the blood-brain barrier, in contrast to ubiquinone. Idebenone performs the same function as ubiquinone - the transfer of electrons in the respiratory chain of mitochondria (Figure 2). Idebenone Helps Improve Cognitive Function $[25,26]$. This medicine can also be used for chronic cerebral ischemia.

For the correction of mitochondrial dysfunctions, drugs are also used that participate in the fat metabolism of mitochondria (carnitine preparations) and carnosine (a dipeptide consisting of two amino acids: beta-alanine and histidine), isolated from muscles in 1903 by Russian scientist V. S. Gulevich [27-29]. Carnosine is synthesized in a number of organs (including the brain) and increases their performance. Dimephosphone, dichloroacetate, citrulline malate - drugs that reduce lactic acidosis, could also be used in cerebral ischemia, given the mitochondrial disorders detected in this pathology [30-32].

Thus, a quantitative cytochemical method for assessing the activity of mitochondrial enzymes in peripheral blood 
lymphocytes, used to assess acute and chronic cerebral ischemia in adult patients, makes it possible to objectively assess the indicators of various types of metabolism in mitochondria and justify the appointment of energotropic therapy.

\section{Conclusion}

Acute and chronic cerebral ischemia is accompanied by impaired mitochondrial function. In the presented study, the quantitative method proposed by A. G. Pearse, to assess the activity of mitochondrial enzymes in peripheral blood lymphocytes, which are referred to as the "enzematic mirror" of tissues. Investigated the activity of four enzymes: succinate dehydrogenase, $\alpha$-glycerophosphate dehydrogenase, glutamate dehydrogenase and lactate dehydrogenase. In stroke (acute cerebral ischemia), blood sampling was performed on the 1st and 7th days. On the first day of the stroke, a compensatory increase in the activity of succinate dehydrogenase was noted, indicating the tension (increased activity) of the II complex of the mitochondrial respiratory chain. These data confirm the necessity of prescribing succinic acid preparations in the acute phase of stroke. At the same time, the dose of the medicine of succinic acid $250 \mathrm{mg}$ per day is not sufficient for patients with increased body weight. The standard dose of this medicine should be increased taking into account the different body weight of patients. In patients with stroke, there was a decrease in the activity of $\alpha$-glycerophosphate dehydrogenase, which is involved in the fat metabolism of mitochondria, which is an indication for the prescription of medicines of carnitine.

In chronic cerebral ischemia, a decrease in indicators of the activity of succinate dehydrogenase and $\alpha$ glycerophosphate dehydrogenase was observed. These data indicate indications for the appointment of idebenone and carnitine along with vasodilator therapy and endovascular thrombectomy in patients with chronic cerebral ischemia.

Thus, the function of mitochondria was determined in patients with acute and chronic cerebral ischemia. Disorders of the II complex in the cycle of the respiratory chain and a violation of fat metabolism were revealed, indicating indications for the appointment of energotropic therapy.

\section{Acknowledgements}

State assignment.

\section{References}

[1] Ten V, Galkin A (2019) Mechanism of Mitochondrial Complex I Damage in Brain Ischemia/Reperfusion Injury. A Hypothesis. Mol Cell Neurosc 100: 103408.

[2] Mironova GD, Pavlik LL, Kirova YI, Belosludtseva NV, Mosentsov AA et al. (2019) Effect of hypoxia on mitochondrial enzymes and ultrastructure in the brain cortex of rats with different tolerance to oxygen shortage. J Bioenerg Biomembr 51: 329-340.
[3] Lukyanova LD, Kirova YI, Germanova EL (2018) The Role of Succinate in Regulation of Immediate HIF-1 $\alpha$ Expression in Hypoxia. Bull Exp Biol Med 164: 298-303.

[4] Lukyanova LD, Kirova YI, Germanova EL (2016) Specific Features of Immediate Expression of Succinate-Dependent Receptor GPR91 in Tissues during Hypoxia. Bull Exp Biol Med 160: 742-7.

[5] Lukyanova LD, Kirova YI (2015) Mitochondria-controlled signaling mechanisms of brain protection in hypoxia. Front Neurosci 1; 9: 320.

[6] Plotnikov DM, Stegmeier MN, Aliev OI (2019) Evaluation of Blood Rheology by Patients With Acute Ischemic Stroke With Mexidol Administration. Zh Nevrol Psikhiatr Im S S Korsakova 119: 76-82.

[7] Yagudina RI, Kulikov AYu, Krylov VA, Solovieva EYu et al. (2019) Pharmacoeconomic Analysis of the Neuroprotective Medicines in the Treatment of Ischemic Strok. Zh Nevrol Psikhiatr Im S S Korsakova 119: 60-68.

[8] Balashova IN, Vanchakova NP, Afanasiev VV, Barantzevich ER, Pugacheva EL et al. (2018) Diagnosis and Treatment of Neurogenic Dysphagia After Acute Ischemic Stroke. Zh Nevrol Psikhiatr Im S S Korsakova 118: 64-69.

[9] Kulai NS, Kovalchuk E Yu (2018) Assessment of the Efficacy of Mexidol in the Combination With Hyperbaric Oxygen in Acute Ischemic Stroke. Zh Nevrol Psikhiatr Im S S Korsakova 118: 69-72.

[10] Sazonov IE, Klementenko TD, Kudinov AA, Avramenko MA, Yarmonov SN (2018) The Use of Cytoflavin in the Acute Period of Hemorrhagic Stroke. Zh Nevrol Psikhiatr Im S S Korsakova 118: 23-26.

[11] Ekusheva EV (2017) Modern Technologies and Prospects of Rehabilitation of Patients After Ischemic Stroke. Zh Nevrol Psikhiatr Im S S Korsakova Affiliations Expand 117: 147-155.

[12] Sarvari S, Moakedi F, Hone E, Simpkins JW, Ren X (2020) Mechanisms in blood-brain barrier opening and metabolismchallenged cerebrovascular ischemia with emphasis on ischemic stroke. Metab Brain Dis 35 (6): 851-868.

[13] Yang JL, Mukda S, Chen SD (2018) Diverse roles of mitochondria in ischemic stroke. Biol 6: 263-275.

[14] Hayakawa K, Esposito E, Wang X, Terasaki Y, Liu Y et al. (2016) Transfer of mitochondria from astrocytes to neurons after stroke. Nature 535: 551-5.

[15] Nguyen H, Zarriello S, Rajani M, Tuazon J, Napoli E et al. (2018) Understanding the Role of Dysfunctional and Healthy Mitochondria in Stroke Pathology and Its Treatment. Int J Mol Sci 21: 2127.

[16] Kotov S V, Sidorova O P, Borodataya E V (2019) Mitochondrial disorders in neuromuscular pathology. Neuromuscular diseases 9: 22-31 (Published in Russian).

[17] Lukyanova LL (2003) Molecular mechanisms of tissue hypoxia and adaptation of the body. Fiziol Journal 49: 17-35. (Published in Russian).

[18] Zhao XY, Lu MH, Yuan DJ, Xu DE, Yao PP et al (2019) Mitochondrial Dysfunction in Neural Injury. Front Neurosci 4: 30.

[19] Lenart J (2017) Mitochondria in brain hypoxia. Postepy Hig Med Dosw (Online) 15: 118-128. 
[20] Du J, Ma M, Zhao Q, Fang L, Chang J et al. (2013) Mitochondrial bioenergetic deficits in the hippocampi of rats with chronic ischemia-induced vascular dementia. Neuroscience 12: 345-52.

[21] Kim AY, Jeong KH, Lee JH, Kang Y, Lee SH et al. (2017) Glutamate dehydrogenase as a neuroprotective target against brain ischemia and reperfusion. Neuroscience 6: 487-500. doi: 10.1016/j.neuroscience.2016.11.007.

[22] Qadri R, Namdeo M, Behari M, Goyal V, Sharma S et al (2018) Alterations in mitochondrial membrane potential in peripheral blood mononuclear cells in Parkinson's Disease: Potential for a novel biomarker. Restor Neurol Neurosci 36: 719-727.

[23] Buyse GM, Voit T, Schara U, Straathof CSM, D'Angelo MG et al. (2017) Treatment effect of idebenone on inspiratory function in patients with Duchenne muscular dystrophy. 52: 508-515.

[24] McDonald CM, Meier T, Voit T, Schara U, Straathof CSM, et al. (2016) Idebenone reduces respiratory complications in patients with Duchenne muscular dystrophy. Neuromuscul Disord 26: 473-80.

[25] Bogolepova AN, Kovalenko EA (2016) Therapy of asthenic and cognitive impairments in chronic cerebral ischemia: the possibilities of idebenone. Doktor Ru 4: 10-13. (Published in Russian).

[26] Qi F-X, Hu Y, Kang L-J, Li P, Gao T-C et al. (2020) Effects of Butyphthalide Combined With Idebenone on Inflammatory
Cytokines and Vascular Endothelial Functions of Patients With Vascular Dementia. J Coll Physicians Surg Pak 30: 2327.

[27] Masuoka N, Yoshimine C, Hori M., Tanaka M., Asada T et al. (2019) Effects of Anserine/Carnosine Supplementation on Mild Cognitive Impairment With APOE4. Nutrients 17: 1626.

[28] Davis C. K., Laud P. J., Bahor Z., Rajanikant G. K., Majid A. Systematic Review and Stratified Meta-Analysis of the Efficacy of Carnosine in Animal Models of Ischemic Stroke // J Cereb Blood Flow Metab. 2016. - Vol. 36 (10): 1686-1694. doi: $10.1177 / 0271678 \times 16658302$.

[29] Kim E-S, Kim D, Nyberg S, Poma A, Cecchin D et al. (2020) Functionalized Polymersomes Enhance the Efficacy of Carnosine in Experimental Stroke. Sci Rep 20: 699.

[30] Arveladze G, Geladze N, Khachapuridze N, Bakhtadze S, N Kapanadze N. (2015) Mitochondrial dysfunction: modern aspects of therapy (review). Georgian Med News 244-245: 7884.

[31] Zhao H, Jao J, Yuan Y, Feng J, Cheng H et al. (2019) Dichloroacetate Stimulates Angiogenesis by Improving Endothelial Precursor Cell Function in an AKT/GSK-3 $\beta / N r f 2$ Dependent Pathway in Vascular Dementia Rats. Front Pharmacol 10: 523.

[32] Wax B, Kavazis AN, Luckett W (2016) Effects of Supplemental Citrulline-Malate Ingestion on blood lactate, cardiovascular dynamics, and resistance exercise performance in trained males. J Diet Suppl 13: 269-82. 